\title{
Creencias de los Formadores de Profesores de Distinto Tipo de Universidades sobre Escuelas en Contextos de Pobreza
}

\author{
Viviana Gómez-Nocetti ${ }^{1}$, Pablo Gutiérrez-Rivera ${ }^{1}$, Alfredo Gaete-Silva ${ }^{1}$ y María L. Marqués-Rosa ${ }^{1}$ \\ (1) Pontificia Universidad Católica de Chile, Campus Villarrica, Región de La Araucanía, Chile. \\ (e.mail:vgomezn@uc.cl; pablogutierrez@uc.cl; agaetes@uc.cl; mamarques@uc.cl;
}

Recibido Jun. 12, 2018; Aceptado Ago. 8, 2018; Versión final Sep. 21, 2018, Publicado Feb. 2018

\begin{abstract}
Resumen
Se exploraron las creencias sobre escuela vulnerable de 22 formadores de profesores de universidades. Estos actores son importantes porque modelan consciente o inconscientemente sus creencias en los estudiantes de pedagogía. Se realizó un estudio cualitativo de construcción de metáforas mediante la metodología Lego ${ }^{\circledR}$ Serious Play ${ }^{\mathrm{TM}}$, las que se analizaron mediante análisis temáticos y de campos semánticos, para luego construir narrativas sobre escuela vulnerable. Se encontraron distintas narrativas en los formadores y diferencias respecto a identidad, factores causales atribuidos y su posible modificabilidad. Se concluye que existen grandes diferencias en sus características profesionales, tales como estudios de postgrado, edad, distribución de género y tipo de contrato. Aquellos formadores de universidades sin requisitos de ingreso se identifican como profesores de aula y poseen creencias prejuiciosas hacia las familias y hacia los hacedores de políticas. Los formadores de la universidad tradicional mencionan contradicciones políticas y sociales, y todos concuerdan en que los profesores deben ser educados en temas como las escuelas vulnerables.
\end{abstract}

Palabras clave: instituciones de educación superior; creencias sobre pobreza; escuela vulnerable; formación inicial de profesores

\section{Teacher Educators' Beliefs of Different Types of Universities about Schools in Poverty Contexts}

\begin{abstract}
The beliefs and concepts of vulnerable school of 22 teacher educators from universities were explored. These actors are important because they consciously or unconsciously model their beliefs and pass them to their student teachers. A qualitative study of the construction of metaphors was carried out using the Lego® Serious Play ${ }^{\mathrm{TM}}$ methodology, which was analyzed through thematic analysis and semantic fields, to then build narratives about vulnerable school. Different narratives were found in the educators and differences regarding: identity, causal factors attributed and their possible modifiability. It is concluded that there are great differences in their professional characteristics such as graduate studies, age, gender distribution and contract type. Those teacher educators without entry requirements identify themselves as classroom teachers and possess prejudiced beliefs towards families and towards policy makers. The trainees of the traditional university mention policies and social contradictions, and all agree on that teaching staff must be educated on concepts related to vulnerable schools.
\end{abstract}

Keywords: higher education institutions; beliefs on poverty; vulnerable school; initial teacher education 


\section{INTRODUCCIÓN}

El modelo neoliberal en que se basa el sistema educativo chileno, ha tenido hondas consecuencias (Villalobos y Quaresma, 2015). El escenario al que nos enfrentamos hoy, está lejos de conformar un sistema articulado y coherente que trabaja en forma sinérgica para resolver los problemas que lo aquejan. Un ejemplo de esto, es que todavía no se ha encontrado la forma en que debiesen dialogar las políticas relativas a las escuelas con aquellas relativas a la formación de profesores o con la investigación sobre educación superior, en pos de alcanzar una educación más equitativa (Darling-Hammond, 2016). En efecto, una de las políticas públicas que más desafía a la formación inicial de profesores, es la actual Ley SEP iniciada el año 2008, la que está orientada a mejorar la equidad en el aprendizaje de los estudiantes más vulnerables, entregando recursos adicionales a las escuelas subvencionadas según la concentración de "niños prioritarios". Esta es una tarea de gran envergadura si consideramos que el promedio nacional de índice de vulnerabilidad de los niños entre 0 y 14 años es de 78,7\% (Observatorio Niñez y Adolescencia, 2016). A casi diez años de su promulgación, esta política ha tenido consecuencias importantes en términos de financiamiento y de hacer más visibles a un gran número de escuelas cuyos estudiantes son, en su mayoría, prioritarios. Estas escuelas, además de ser sometidas a fuertes demandas por mostrar resultados en el corto y mediano plazo, han pasado a ser etiquetadas popularmente como "escuelas vulnerables", lo que ha implicado el éxodo de alumnos noprioritarios a escuelas más selectivas (Villalobos y Quaresma, 2015). Por otra parte, los resultados siguen siendo influenciados por el nivel socioeconómico, el que explica la gran brecha de rendimiento entre alumnos de NSE bajo y NSE alto.

En el extremo contrario, el mercado de educación superior ha promovido la existencia de una oferta desregulada de programas de formación de profesores, que difieren en calidad y prestigio (Meckes y Bascopé, 2012; Ávalos, 2014), incluso en lo que respecta a su cuerpo académico (Montenegro, 2016). Dado este contexto, asumir la demanda de formar profesores que aseguren mejores logros de aprendizaje en los niños objeto de esta política, requiere reflexionar sobre cuáles son los nudos a los que debe apuntar la formación. Proponemos que uno de estos debiera ser abordar el efecto que está teniendo en los profesores el uso de las categorías y clasificaciones (Bourdieu, 1997) propias de la Ley SEP. Esto porque la literatura señala que los profesores en servicio presentan creencias estereotipadas sobre los alumnos provenientes de sectores de pobreza (por ejemplo, ver Apablaza, 2014) y que estas creencias influyen en la calidad de la educación que ofrecen los profesores a los niños en desventaja (Rjosk et al., 2014), ya que cuando aplican la etiqueta "pobre" a un niño, la usan como excusa para explicar por qué este fracasa (Ullucci y Howard, 2015). Sin embargo, algo importante de destacar aquí, es que estas creencias no solo aparecen entre los profesores de aula. En un estudio cuantitativo a nivel nacional, nuestro equipo de investigación constató hallazgos previos respecto a los profesores en servicio, pero también encontró que entre formadores de profesores y estudiantes de pedagogía aparecían creencias ubicadas en un punto intermedio entre creencias complejas y creencias estereotipadas, con una importante dispersión entre participantes (Gómez, 2017). Además, la literatura señala que las creencias de los profesores no cambian porque estas no fueron abordadas sistemáticamente en los programas de formación universitaria y se mantienen implícitas hasta que enfrentan su propia práctica pedagógica (Joram, 2007; Pajares, 1992). Sin embargo, hay una razón para tener esperanzas pues Levin y He (2008) encontraron que los programas de formación podrían tener hasta un 30\% de influencia sobre las creencias explícitas que los futuros profesores desarrollan durante su preparación, por lo que es posible intervenir intencionadamente para producir cambios en las creencias.

Dado el particular escenario que hemos esbozado, en este artículo ahondaremos en las creencias de los formadores de profesores en una muestra de formadores de universidades reguladas y desreguladas. Nos interesa este actor debido al importante papel de modelamiento que posee frente a sus estudiantes, lo que podría estar incidiendo en la mantención de ciertas creencias que consolidan el fracaso de los niños prioritarios.

\section{EFECTOS DE LA POLÍTICA NEOLIBERAL}

Además de sus efectos en el sistema escolar, la educación de mercado, producto del modelo económico neoliberal chileno iniciado en los años 80 , ha significado importantes cambios para la educación superior. Entre los más importantes observamos que, de las 14 universidades en esa década, actualmente existen 59 instituciones, 21 de las cuales no poseen requisitos de ingreso y tienen manifiestos fines de lucro (Bruner, 2015). Este último tipo de universidades se caracteriza por su baja calidad, lo que se observa en indicadores tales como la acreditación, puesto que poseen en promedio 1,65 años, en comparación con las universidades tradicionales que poseen entre 4,5 y 5,4 años. Lo paradójico es que, a pesar de este antecedente, atraen cada vez a más estudiantes. Al parecer, la facilidad para ingresar a ellas y el costo de sus carreras suelen atraer a los estudiantes menos exitosos del sistema educativo, pues pueden valer hasta dos millones de pesos menos (unos USD 3.000 aproximadamente) por año de estudios, comparado con aranceles que llegan al doble en universidades tradicionales y hasta el cuádruple en las nuevas universidades de élite (Consejo 
Nacional de Educación, 2017). Es así como el 30\% de estudiantes egresados de colegios municipales, el $62.4 \%$ de quienes estudiaron en colegios subvencionados y un $7.4 \%$ de quienes estudiaron en colegios particulares, se matricula en las instituciones más desreguladas (Consejo Nacional de Educación, 2017).

Por otra parte, un rasgo que no ha sido polemizado es que estas instituciones poseen plantas académicas de hasta un $99 \%$ de docentes contratados por hora, a quienes no se les exige estudios de post-grado ni poseer experiencia previa en docencia universitaria. En cambio, las universidades tradicionales poseen entre $39 \%$ y 45\% de profesores por hora, los que sí poseen estudios de postgrado (Consejo Nacional de Educación, 2017). Esto mismo ocurre en las instituciones formadoras de profesores, pues un estudio realizado por Montenegro (2016) en universidades chilenas, muestra que existe una gran heterogeneidad de perfiles entre los formadores de profesores y que la mayoría posee solo experiencia áulica en escuelas. Además, encontró que la mayor parte de las instituciones no realiza programas de inducción cuando estos ingresan a la academia, no incentiva la investigación ni entrega horas para el trabajo en equipo.

Lo anterior es importante, pues el profesor de aula tiene un papel distinto al del formador de profesores y la transición de uno al otro no es una tarea simple, debido al cambio en la naturaleza y rol de la enseñanza y las expectativas de la academia (Loughran, 2014). Murray y Male (2005) conceptualizan a los formadores de profesores como aquellos académicos que se han movido desde una posición de profesionales de primer orden, es decir, cuya experiencia proviene de la enseñanza como profesores de aula en el sistema escolar, a ser profesionales de segundo orden, cuyo papel es inducir a sus estudiantes en prácticas y discursos propios de la enseñanza en contextos escolares como de la formación docente. Sin embargo, el conocimiento del profesional de primer orden a menudo es tácito, permeado por la práctica y por una forma individual de comprender la enseñanza y el aprendizaje, que además está saturado de valores, creencias y biografías personales. No obstante, Murray y Male (2005) señalan que, con un adecuado sistema de inducción en las escuelas de formación, la adquisición de la nueva identidad como profesional de segundo orden, podría demorar entre 2 y 3 años. Martinez (2008) muestra los desafíos específicos que debe enfrentar un profesor para transformarse en formador de profesores, por ejemplo, debe enseñar a nuevos estudiantes, que son adultos, no niños y, además, debe modelar lo que enseña, lo que demanda una sofisticada metacognición, pues no solo debe "hacer" sino también comentar, explicar y justificar sus prácticas docentes. Por esto, no se trata transferir las habilidades docentes a un contexto diferente, este paso requiere un proceso de formación (Loughran, 2017). En la actualidad, existen programas formales para formadores de profesores que se centran tanto en su identidad profesional como en sus actitudes y creencias, en Holanda (Boei et al., 2015), en Israel (Hadar y Brody, 2016) o en países del sudeste asiático (Borg et al., 2018). Sin embargo, estos aún son muy incipientes en Latinoamérica y en Chile (para más detalle, ver González-Vallejos, 2018).

Ahora bien, estos hallazgos, debieran hacernos pensar sobre la posibilidad de que muchos de los formadores de profesores, que actúan como profesores de aula, puedan estar modelando implícitamente en sus estudiantes una visión negativa de la pobreza y de los pobres (Gorski, 2016). Esto debiera preocuparnos especialmente porque una vez egresados de la formación inicial, estos profesores irán a las escuelas a perpetuar el círculo del fracaso (Hughes, 2010). Ya que la investigación sobre los formadores de profesores en Chile todavía es escasa (González-Vallejos, 2018; Montenegro, 2016), este artículo pretende aportar a este campo, explorando las creencias sobre pobreza de formadores de profesores de universidades con y sin requisitos de ingreso.

\section{FORMACIÓN INICIAL DOCENTE}

La matrícula en las carreras de pedagogía también ha mostrado un crecimiento desregulado a partir del año 2003, especialmente en las instituciones privadas con menos o sin ningún requisito de ingreso (Ávalos, 2014). Un análisis de regresión realizado por Pedraja et al. (2012) muestra una relación positiva y directa entre los años de acreditación de las carreras y el puntaje obtenido en la prueba INICIA (este instrumento, que se aplicó desde el año 2008 al 2014 a los egresados de Pedagogía, medía el nivel de logro de los estándares disciplinares y pedagógicos necesarios para ser profesor) por sus egresados. Esto provoca una nueva y perversa relación positiva y directa, entre el estatus de la institución formadora y el estatus de las escuelas a las que acceden sus egresados, ya que los profesores que enseñan en escuelas más pobres suelen venir de los mismos contextos educativos que sus alumnos, tal como muestran diversos estudios como los realizados por Meckes y Bascopé (2012), Rivero (2015), Puga et al. (2015) y Ortúzar et al. (2016). Por ejemplo, Meckes y Bascopé (2012), al estudiar una muestra de 1.927 (48\%) graduados el año 2008, encontraron que la mayoría de quienes trabajaban en escuelas subsidiadas el año 2010, habían estudiado en escuelas subsidiadas $(51,2 \%)$ y que el $61 \%$ de ellos tenía padres sin educación terciaria. A su vez, los egresados de colegios privados pagados tenían menor probabilidad de trabajar en estas escuelas que los egresados que provenían de escuelas públicas o privadas subvencionadas. Además, a mayor puntaje de salida, mayor probabilidad tenían de encontrar trabajo en escuelas de mayor estatus socioeconómico y que quienes estudiaron en programas nocturnos a tiempo parcial, tenían un 50\% de probabilidad de trabajar en escuelas de bajo NSE y 
con menor logro en las pruebas estandarizadas en lenguaje y matemáticas. Rivero (2015) estudió a 79.418 profesores de enseñanza básica revisando la distribución de profesores de calidad a lo largo de las escuelas en el país (según grado de selectividad de la institución de origen, el grado obtenido y la especialidad, la duración del programa de formación, la experiencia docente y el logro en pruebas de evaluación docente). Tras una serie de interesantes análisis, el estudio concluyó que "las escuelas rurales y públicas tienden a emplear mayores proporciones de profesores menos calificados y los estudiantes pobres también tienden a ser enseñados por profesores menos calificados. Además, los estudiantes con más bajo rendimiento tienen más probabilidad de ser matriculados en escuelas que emplean a los profesores con menos habilidades. Por ejemplo, solo el $20 \%$ de los profesores que trabajan en escuelas de bajos ingresos estudiaron en un programa de formación con una duración de 10 o más semestres, comparado con el $44 \%$ de profesores que trabajan en escuelas de altos ingresos" (Rivero, 2015, p.19).

Esto coincide con Puga et al. (2015) quienes, tras estudiar a más de 5.000 profesores de educación básica, compararon los índices de origen social, formación inicial y de condiciones laborales y encontraron que "existe un efecto significativo y muy considerable $(0,357)$ del origen social de los profesores en el acceso a una educación más exclusiva en instituciones más selectivas" (p. 77). Esto quiere decir, que un profesor de bajo índice de origen social tiene un $67 \%$ de probabilidades de enseñar en una escuela municipal. Los autores concluyen que el fenómeno de la segregación ocurre en todos sus procesos, es decir, que el origen social de los docentes determina el tipo de establecimiento escolar en que estudiará, así mismo determina el tipo de formación inicial al que accederá y posteriormente, al tipo de escuela en que se desempeñará y al logro de sus estudiantes en el área de lenguaje. Un antecedente adicional es entregado por Ortúzar et al., (2016), quienes se interesaron por estudiar los criterios aplicados en los procesos de contratación en los distintos los tipos de escuela, revelando que existen nudos problemáticos importantes que explican esta desigual distribución de profesores y entregan algunas sugerencias para superarlos. Pero, ¿por qué esto es un problema? La respuesta es que quienes egresan de universidades menos selectivas, probablemente han recibido una formación profesional más débil, la que, sumada a su propia debilidad académica derivada de su origen sociocultural y educativo, ofrecerá un escenario que difícilmente revertirá las precarias condiciones educativas a las que acceden los niños con mayor desventaja en el país (Meckes y Bascopé, 2012), incluso, Puga et al. (2015) especulan que es posible que los bajos resultados de los alumnos sea producto del capital cultural de los profesores.

Bourdieu (1997) señala que es el sistema escolar el que, mediante una serie de operaciones de selección, separa a quienes poseen capital cultural heredado de quienes carecen de él, y con esto, tiende a mantener las diferencias sociales preexistentes. Los profesores no son inmunes a la influencia de los estereotipos y, a través de ciertas claves comportamentales y de la evaluación escolar, informan a sus alumnos acerca de sus expectativas e influyen sobre las interpretaciones atribucionales de sus propias capacidades académicas (Georgiou, 2008). Para Bourdieu, el habitus del profesor contribuye a la reproducción de la inequidad social en la vida cotidiana de la escuela. Pero este no es un proceso consciente, pues las actitudes y clasificaciones del habitus parecen funcionar sin reflexión ni consciencia, para expresarse en forma automática. Estos esquemas de clasificación estarían expresando las divisiones sociales internalizadas por los profesores, las que adquieren a partir de las experiencias y hábitos de su medio social, los que son puestos en práctica de forma no muy diferentes a un principio universal o la escritura personal. Esto significa que todos los profesores han adquirido, implícitamente, categorías sociales que han sido incorporadas como naturales. En el caso de los futuros profesores provenientes de sectores de pobreza habrá mayor probabilidad de transmitir visiones negativas sobre la pobreza, porque ellos no solo han incorporado estas categorías como normales, sino que han asumido el lugar en la sociedad que les ha sido asignado por medio una serie de prácticas sociales que transmiten el orden social. Esto ocurre principalmente como producto de la escolarización, ya que como Bourdieu (1999) señala, la escuela tiene como función dotar a la población de las categorías que permitirán la construcción de una nación con el mismo sentido común. Este es un fundamento de por qué los programas de formación inicial de profesores deben explícitamente abordar estas creencias para cortar las raíces profundas que hacen que, generación tras generación, los profesores realicen prácticas que le digan a los pobres cuál es el lugar que les corresponde en la sociedad.

Este estudio sostiene una visión las creencias como sistemas. Estas se entienden como "juicios individuales acerca de la verdad o falsedad de una proposición, un juicio que puede ser solo inferido desde una comprensión colectiva de lo que los seres humanos dicen, intentan y hacen" (Pajares, 1992, p. 316). Además, tienen una función adaptativa y se organizan en sistemas, por lo que ayudan a definir y entender al mundo y a sí mismo. Por su naturaleza afectiva, valorativa y episódica, las creencias se transforman en verdaderos filtros a través de los cuales se interpretan los nuevos fenómenos. Es decir, la estructura de creencias redefine, distorsiona o remodela el pensamiento y el procesamiento de la información (Fives y Buehl, 2012). Por otra parte, algunas creencias son más indiscutibles que otras. Incluso, se priorizan en función de sus conexiones o relación con otras creencias -en tanto sistemas- u otras estructuras cognitivas y afectivas, moderadas por la situación (Fives y Buehl, 2012). 
Lamentablemente, a nivel nacional, no existen suficientes estudios de las creencias de los formadores de profesores (ver González-Vallejos, 2018). Esto relevante porque Levin y He (2008) señalan que en los programas de formación inicial hay dos fuentes que influyen poderosamente en las creencias de los estudiantes de pedagogía. Estos reconocieron que un 31\% provenía de los cursos, lecturas, teorías e ideas de sus formadores a los que se han enfrentado y un $35 \%$ de haber observado y participado en prácticas pedagógicas en las escuelas. La literatura señala que los programas de formación de profesores no tienen impacto en las creencias de los futuros profesores porque no las abordan directamente (Joram, 2007; Pajares, 1992). Como resultado, estos pondrán en juego sus creencias para comprender su rol como enseñante, confirmarán sus expectativas sobre sus estudiantes pobres y reforzarán creencias y prácticas de enseñanza y aprendizaje que reproducirán y explicarán los bajos resultados de sus alumnos (Rjosk et al., 2014). Una vez egresados de la formación inicial, estos profesores irán a las escuelas a perpetuar este círculo del fracaso (Hughes, 2010).

\section{METODOLOGÍA}

El estudio tuvo un diseño cualitativo, en la línea del integracionismo teórico (Gaete y Gaete, 2014, 2015; Cook y Reichardt, 2005). Desde esta perspectiva, supuestos epistémicos provenientes de distintos programas de investigación pueden y deben combinarse o preferirse según los requerimientos del problema en cuestión, de tal modo que "a la hora de elegir un método, (carece) de importancia la posición paradigmática (...) La elección del método de investigación debe depender también, al menos en parte, de las exigencias de la situación de investigación de que se trate" (p. 37). De hecho, a nivel epistemológico existen sólidas razones para no abordar los problemas desde un solo paradigma o programa de investigación (Gaete y Gaete, 2014, 2015). También hay buenas razones para evitar el uso de metodologías cuantitativas cuando el problema en cuestión son las creencias pedagógicas (véase Gaete, Gómez y Benavides, 2018).

En este estudio hemos recurrido a una versión de la metodología Lego SeriousPlay (LSP), adaptada por Gutiérrez (2015) para ser usada en contextos educativos. Se trata de una excelente herramienta para representar las creencias más implícitas en los formadores de profesores sobre las escuelas vulnerables, ya que, a nuestro juicio, permite controlar algunas de las condiciones bajo las cuales las estrategias habitualmente empleadas en la investigación sobre creencias tienden a fallar, especialmente la "condición de expresabilidad" (véase Gaete, Gómez y Benavides, 2018).

\section{Procedimiento}

Este estudio proviene de un programa de investigación mayor relacionado con el cambio conceptual de las creencias sobre escuelas en contextos de pobreza, que sostienen estudiantes de pedagogía, profesores en servicio y formadores de profesores de distintas universidades y escuelas de las tres regiones en las que se realiza este programa, a saber, Región Metropolitana, Región de La Araucanía y Región de Los Lagos. La primera fase fue cuantitativa y mostró la existencia de visiones estereotipadas, intermedias y complejas, que presentaron estos tres tipos de actores educativos indistintamente (ver Gómez, 2017). La segunda fase consistió en la constitución de "Grupos de Indagación" a través de la convocatoria a participar en una reflexión colegiada entre formadores de profesores. El objetivo de estas sesiones era evaluar el potencial para producir cambio en las creencias de distintas estrategias de reflexión tales como juegos de tablero, casos y la estrategia Lego® SeriousPlay® (LSP). Se realizaron cuatro Grupos de Indagación; dos grupos trabajaron con la estrategia LSP y los otros dos grupos utilizaron casos y juegos de tablero. La invitación a los Grupos de Indagación fue abierta y voluntaria, intentando abarcar el mayor número de formadores posible, por lo tanto, el resultado fue que los dos grupos informados en este artículo, tuvieron un tamaño diferente. Para tener representaciones más o menos equivalentes en número, se decidió que en el caso de la universidad privada sin requisitos de ingreso se formaran siete grupos, dada la gran cantidad de participantes que aceptaron nuestra invitación.

\section{Preguntas y objetivos del estudio}

En la fase cuantitativa previa, se encontró que los formadores de profesores poseían creencias distintas a las esperadas. Es decir, un 36\% de formadores de profesores presentaba una visión estereotipada sobre la escuela vulnerable, un 52\% presentaba visiones intermedias y solamente un 12\% presentó una visión compleja al respecto; además, se encontraron diferencias significativas a favor de los formadores que poseen grado de Doctor (ver Gómez, 2017). A partir de esta constatación, surgieron nuevas preguntas ¿existen diferencias en las creencias sobre escuela vulnerables según el tipo de institución a la que pertenecen los formadores? ¿Cuáles son esas creencias? ¿Qué características tienen los cuerpos académicos de los programas de formación en estas distintas instituciones? Los objetivos de este estudio fueron 1) caracterizar a los formadores de profesores de distintos tipos de instituciones formadoras de profesores en la actualidad 
(tradicional y privada sin requisitos de ingreso) y, 2) explorar en profundidad las creencias sobre escuela vulnerable de formadores de ambos tipos de universidades.

\section{Participantes}

En este estudio participaron 22 formadores de profesores, 15 de los cuales trabajaban universidades privadas sin requisitos de ingreso (quienes se organizaron en 7 grupos) y 7 formadores de profesores de una universidad privada tradicional, todos pertenecientes a la zona sur de Chile. Para asegurar el cumplimiento de los requisitos éticos de la investigación, estos leyeron y firmaron un consentimiento informado, antes de aceptar su participación en estas sesiones. Para asegurar la privacidad de la información de los participantes, se ha denominado a las universidades de forma genérica como "universidad tradicional" y "universidad privada sin requisitos de ingreso".

Como podemos observar en la tabla 1, la edad promedio de los formadores en la universidad tradicional fue de 44.1 años y en la universidad privada sin requisitos de ingreso fue de 42.9 años. En ambos casos, predomina el género femenino por sobre el masculino. Otro dato importante, es que ambos grupos difieren considerablemente en la proporción de profesionales con postgrado. En la universidad tradicional todos los formadores cuentan con postgrado y más de la mitad tiene grado de doctor. En cambio, entre los formadores de la universidad sin requisitos de ingreso, la mayor proporción es la que posee solo licenciatura en educación. En cuanto a postgrado, solo se observan estudios de magister. Las jornadas de trabajo también son distintas, predominando los contratos por hora por sobre la jornada completa en la universidad sin requisito, lo que no existe en la universidad tradicional.

Como se observa en la tabla 1, mientras los formadores de profesores de las universidades sin requisitos de ingreso poseían principalmente solo licenciatura y dictaban uno o dos cursos en sus universidades, los formadores de profesores de la universidad tradicional eran en su mayoría doctores de jornada completa.

Tabla 1: Descriptivos de la muestra de formadores de profesores participantes según grado académico, tiempo de contratación, edad y género.

\begin{tabular}{|c|c|c|c|c|}
\hline \multirow[t]{2}{*}{ Característica/tipo formador } & \multicolumn{2}{|c|}{ Universidad tradicional } & \multicolumn{2}{|c|}{$\begin{array}{l}\text { Universidad privada sin } \\
\text { requisitos de ingreso }\end{array}$} \\
\hline & $N$ & $\%$ & $N$ & $\%$ \\
\hline \multicolumn{5}{|l|}{ Grado académico: } \\
\hline - Licenciado & 0 & - & 8 & $53.3 \%$ \\
\hline - Magister & 2 & $40 \%$ & 7 & $46.7 \%$ \\
\hline - Doctor & 5 & $60 \%$ & 0 & - \\
\hline \multicolumn{5}{|l|}{ Modalidad: } \\
\hline - Profesor hora & 0 & - & 10 & $66.7 \%$ \\
\hline - Media jornada & 1 & $14.3 \%$ & 1 & $6.7 \%$ \\
\hline - Jornada completa & 6 & $85.7 \%$ & 4 & $26.7 \%$ \\
\hline \multicolumn{5}{|l|}{ Edad: } \\
\hline 25-32 años & 0 & - & 3 & $20 \%$ \\
\hline 33-40 años & 3 & $42.8 \%$ & 4 & $26.7 \%$ \\
\hline 41-48 años & 1 & $14.3 \%$ & 3 & $20 \%$ \\
\hline 49-56 años & 3 & $42.8 \%$ & 4 & $26.7 \%$ \\
\hline 57-65 años & 0 & - & 0 & - \\
\hline Más de 65 años & 0 & - & 1 & $6.7 \%$ \\
\hline \multicolumn{5}{|l|}{ Género: } \\
\hline Femenino & 4 & $57.1 \%$ & 11 & $73.3 \%$ \\
\hline Masculino & 3 & $42.8 \%$ & 4 & $26.7 \%$ \\
\hline
\end{tabular}

Instrumento y materiales

Se utilizó la estrategia Lego® SeriousPlay® (LSP) (adaptada por Gutiérrez, 2015), ya que permite no solo explicitar el pensamiento sin sesgos y, además, permite la movilidad del mismo. La literatura especializada revela que estos modelos representan, a través de imágenes externas tridimensionales de la realidad, los pensamientos e ideas internas de las personas (James, 2013) y su efectividad está relacionada con principios implícitos que ocurren durante la experiencia. La primera característica de LSP es el aprendizaje a través del juego y la expresión personal y colectiva usando la visualización y la construcción de modelos LEGO o 
metáforas, integrando el pensamiento con las manos. La dinámica se caracteriza porque otorga tiempo a las personas para reflexionar y articular sus pensamientos antes de socializar los significados de sus modelos 0 metáforas (Kristiansen y Rasmussen, 2014).

El procedimiento completo de la estrategia Lego® SeriousPlay ${ }^{\circledR}$ (adaptada por Gutiérrez, 2015), consiste en que los participantes debían 1) construir representaciones o modelos individuales de un concepto dado, con piezas LEGO, 2) socializar sus modelos con el resto de participantes, 3) votar por los componentes de los modelos de los otros participantes que mejor aportan a comprender el fenómeno representado, 4) construir colaborativamente una representación conjunta, negociando los significados y elaborando una narrativa compartida, 5) tensionar sus representaciones a través de la exposición de las metáforas de tensionadores experimentados, 6) votar por los componentes de los tensionadores experimentados que enriquezcan el modelo compartido e incorporarlos a esta representación, 7) construir representaciones de distintos agentes o factores que influyen en el fenómeno dado, 8) establecer conexiones entre los agentes y el fenómeno, utilizando conectores de LSP flexibles o rígidos en función del tipo de relación entre ellos. En síntesis, la estrategia LSP permite que los participantes inicien su reflexión a partir de modelos individuales, hasta generar modelos compartidos, para elaborar luego un sistema con agentes conectados. Mediante este proceso ellos pueden construir y re-construir sus creencias materializadas en modelos LEGO, desde representaciones individuales centradas en escuelas a una visión sistémica colectiva del sistema educativo y su complejidad.

Si bien el objetivo de las sesiones de Grupos de Indagación iba orientado al cambio de creencias mediante LSP, los resultados que se presentan en este artículo corresponden a los pasos 1 y 2 , debido a que estos reflejan las creencias iniciales de los sujetos. En el paso 1 se les pidió construir metáforas tridimensionales con piezas Lego, acerca de 1) la escuela vulnerable, y 2) los niños que asisten a estas escuelas y sus familias. En el paso 2, cada participante socializó sus metáforas, explicando los significados atribuidos a cada componente de su construcción. Las sesiones fueron grabadas en video y audio y luego fueron transcritas para su posterior análisis. Cabe señalar que el término "vulnerable" se utilizó en lugar de "pobre" o "bajo nivel socioeconómico" debido a que es la forma en que se denomina comúnmente a las escuelas con alta concentración de niños prioritarios. De esta manera se podrían capturar mejor los significados atribuidos a estas escuelas, sin que medie algún sesgo de deseabilidad social.

En cuanto a materiales, los participantes contaron con numerosas y variadas piezas del set Lego® SeriousPlay $\AA^{\circledR}$, el que contiene los tradicionales ladrillos, además de numerosas figuras humanas ensamblables; animales (perros, tiburones, arañas); complementos (teléfonos, maletines, sobreros, coronas, escobas, picotas, etc.); ruedas, engranajes, talarañas, rejas y diverso tipo de conectores (cuerdas, cadenas tubos, piezas articuladas, etc.). Todo el material estaba dispuesto en un mesón de libre acceso, para que cada formador construyera libremente su metáfora sobre una base también de la línea Lego.

\section{Análisis}

Las metáforas fueron procesadas mediante análisis temático y análisis de campos semánticos, con el fin de configurar las creencias de los dos grupos de formadores de profesores, en un intento de detectar la importancia y el significado del discurso de los grupos dentro de un contexto socio-cultural, explicitando la perspectiva de los sujetos en el marco de su discurso global (Serbia, 2007). En primer lugar, se estableció el campo semántico a estudiar, a saber, la "escuela vulnerable" y el corpus a analizar: "las transcripciones del discurso de las representaciones construidas sobre escuela vulnerable por los formadores de ambos tipos de universidades". A continuación, tres evaluadores leyeron las transcripciones y levantaron las categorías que emergieron de las explicaciones de las metáforas sobre escuela vulnerable y sobre niños y familias que asisten a estas escuelas (por ejemplo "autoridades", "profesores", "familias", etc.), y seleccionaron los extractos textuales que las ejemplificaban. Tras la comparación y establecimiento de las coincidencias, se unieron los extractos pertenecientes a cada categoría y estas se renombraron usando nuevas etiquetas para nominar cada núcleo semántico (por ejemplo, "autoridades incompetentes", "políticas públicas ineficientes") incluido en el campo semántico "escuela vulnerable". Finalmente, se discutieron los significados atribuidos por los participantes y se establecieron las que podrían ser las narrativas sobre la escuela vulnerable subyacentes.

\section{RESULTADOS}

A continuación, presentaremos las narrativas de los formadores de ambos tipos de universidad. En primer lugar, la de la universidad tradicional y luego, la de la universidad sin requisitos de ingreso.

\section{La escuela vulnerable según los formadores de la universidad tradicional}

En el plano social destacan la discriminación y el prejuicio que pesan sobre los niños más pobres: se espera poco de ellos, por lo tanto, se les ofrecen menos oportunidades. Por otra parte, algunos formadores 
cuestionan la existencia de una escuela vulnerable 'per se' pues sería el producto de un sistema político, social, económico e histórico. Sería el resultado de las políticas públicas neoliberales que, por medio de la clasificación de los niños, busca mecanismos "objetivos" para distribuir nuevos fondos públicos para apoyar a las escuelas según la concentración de problemas. Estos criterios toman forma de etiquetas -"niños prioritarios", "sectores vulnerables", "índice de vulnerabilidad"- que construyen "escuelas vulnerables." La tabla 2 presenta los campos semánticos de los formadores de profesores de la universidad tradicional.

Tabla 2: Temáticas y campos semánticos de los formadores de profesores de la universidad tradicional.

\begin{tabular}{|c|c|}
\hline Ideas clave & Campos semánticos \\
\hline Sistema vulnerador & $\begin{array}{l}\text { "son camuflados por el estereotipo", "son sometidos a experiencias de aprendizaje } \\
\text { empobrecidas", "esto se exacerba con políticas de subvención por número de niños prioritarios", } \\
\text { "entrega más dinero por clasificar y segregar aún más", "sufren el prejuicio sobre sus } \\
\text { posibilidades futuras", "sus contextos de origen son estereotipados, vistos como demonios", "el } \\
\text { estereotipo disminuye su potencial y los vulnera", "el sistema los vulnera", "son segregados por } \\
\text { las otras familias y escuelas", "están encerrados en experiencias de aprendizaje que no } \\
\text { desarrollan todo su potencial", "necesitan mayor cuidado de adultos", "hay niños con capacidad } \\
\text { de adaptarse a cualquier situación, que son los que tienen éxito", "otros no están tan adaptados } \\
\text { y sufren, pero no tienen otras posibilidades". }\end{array}$ \\
\hline $\begin{array}{l}\text { Políticas públicas } \\
\text { segregadoras }\end{array}$ & $\begin{array}{l}\text { "etiqueta puesta desde fuera por el sistema", "constructo creado", "usado por los medios", } \\
\text { "resultado de una estructura social, política y económica que segrega en todo sentido", "entrega } \\
\text { dinero a partir de etiquetas", "leyes que sobre-supervisan a escuelas del sistema público", "leyes } \\
\text { que se superponen, clasificando y segregando para otorgar financiamiento", "incrementan el } \\
\text { problema del prejuicio", "presenta una realidad que determina el camino de los más pobres", } \\
\text { "incluso la Ley SEP los vulnera, aunque esté orientada a mejorar los aprendizajes", } \\
\text { "vulnerabilidad es interpretada desde nociones psicosociales, distorsionando la orientación } \\
\text { pedagógica de la Ley SEP", "aunque hay leyes para incluir, estos niños no son incorporados". }\end{array}$ \\
\hline $\begin{array}{l}\text { Espacio educativo } \\
\text { inadecuado }\end{array}$ & $\begin{array}{l}\text { "enrejado", "sin acceso al exterior", "cerrado", "ajeno al mundo", "sucio", "descuidado", "poca } \\
\text { vegetación", "con restricciones para moverse libremente", "sin conexión con la comunidad", } \\
\text { "desconectada del mundo cultural, social y natural". }\end{array}$ \\
\hline $\begin{array}{l}\text { Profesor mal } \\
\text { preparado }\end{array}$ & $\begin{array}{l}\text { "hace niños miopes, no los deja pensar", "se preocupa del uniforme y de que estén tranquilos, } \\
\text { no del aprendizaje", "no cree en los niños, los restringe", "espera un aprendizaje homogéneo", } \\
\text { "se siente dueño de su clase", "no es educador sino capacitador", "trabaja aislado", "toma malas } \\
\text { decisiones sin aprovechar posibilidades del entorno". }\end{array}$ \\
\hline $\begin{array}{l}\text { Profesor con } \\
\text { prejuicios }\end{array}$ & $\begin{array}{l}\text { "ve al niño con carencias, que no tiene nada, que no puede aprender", "ve a la familia como } \\
\text { caótica, que no apoya a sus hijos", "solo ve barreras que no permiten ver el potencial de los } \\
\text { niños", "pasa a llevar a los niños", "ve negativamente a los niños, no confía en ellos", "no está } \\
\text { comprometido", "está abrumado por la problemática creada por la estructura social y política, } \\
\text { cruzada con sus prejuicios". }\end{array}$ \\
\hline Los niños & $\begin{array}{l}\text { "con abundancia de potencial", "son como todos los niños", "no hay mucha diferencia", "son } \\
\text { diversos porque cada ser humano es diferente", "aprenden haciendo, jugando, no escuchando } \\
\text { solamente", "tienen un conocimiento, una historia previa", "son trabajadores, que miran el mundo } \\
\text { desde el jugar y la imaginación", "conectados naturalmente con el medio, es su esencia", } \\
\text { "aventureros, toman riesgos, exploran, van más allá", "resilientes, guerreros", "quieren dar la } \\
\text { pelea, quieren estudiar". }\end{array}$ \\
\hline
\end{tabular}

Para todo este grupo de formadores, el profesor aparece como el actor más importante, pues es quien hace posible la reproducción de la desigualdad, vulnerando directamente a los niños a través de su acción pedagógica cotidiana. Al no estar preparado para trabajar con estos niños y al detentar prejuicios respecto a su contexto, su trabajo resulta ineficaz y nefasto porque busca estandarizar a estos niños a través del disciplinamiento y la entrega de herramientas mínimas. Esto se traduce en un ambiente poco estimulante, cerrado, limitado, empobrecido en todo sentido. Así, profesores y niños parecen hundirse en un espacio que atenta contra su dignidad, sin saberlo ni entenderlo. Los niños, que no son distintos al resto de los niños, van padeciendo la vulnerabilidad, producto de un sistema que no sabe cómo resolver las desigualdades alimentadas por el prejuicio.

\section{La escuela vulnerable según los formadores de universidad tradicional sin requisitos de ingreso}

Este grupo de formadores habla principalmente desde su identidad de profesor de aula, puesto que en varias oportunidades refieren al "nosotros." Con esto en mente, podemos destacar dos núcleos críticos que, para ellos, explican la complejidad ('dificultad') de trabajar en estas escuelas: las autoridades incompetentes y las familias inadecuadas. La tabla 3 presenta los campos semánticos a los cuales se refiere este grupo de formadores de profesores. 
Tabla 3: Temáticas y campos semánticos de los formadores de profesores de la universidad privada sin requisitos de ingreso.

\begin{tabular}{|c|c|}
\hline Ideas clave & Campos semánticos \\
\hline $\begin{array}{l}\text { Autoridades } \\
\text { incompetentes }\end{array}$ & $\begin{array}{l}\text { "poder que camina lento", "que pisa y arrasa", "entes de la educación que están apartados, } \\
\text { mirando desde fuera o desde el centro, pero no están comunicados" } \\
\text { "no hay dirección, llega y aplasta no más", "los que están en el poder ni siquiera saben de } \\
\text { educación", "no han estado en un colegio vulnerable", "está mal pelado el chancho", "el DAEM } \\
\text { no ha ido a colegios donde el desayuno es malo", "a nivel regional o comunal carecen de } \\
\text { conocimientos, pero nos están dirigiendo, están tomando las decisiones". }\end{array}$ \\
\hline $\begin{array}{l}\text { Políticas públicas } \\
\text { ineficaces }\end{array}$ & $\begin{array}{l}\text { "hay muchos recursos financieros, pero muchos no se usan", "no hay un propósito", "no se } \\
\text { concreta nada", "políticas que no se aplican correctamente, porque no se puede", "se arrojan } \\
\text { millones de pesos al sistema para nada". }\end{array}$ \\
\hline $\begin{array}{l}\text { Profesor mal } \\
\text { preparado (al } \\
\text { hablar de la } \\
\text { escuela vulnerable) }\end{array}$ & $\begin{array}{l}\text { "está desactualizado, pero no ve que está mal", "si no cuenta con las herramientas, podría } \\
\text { producir la vulnerabilidad", "tiene un rol fundamental, pero él mismo impide el logro", "tiene pocas } \\
\text { expectativas de sus alumnos", "no usa los materiales porque no confía en que sus alumnos } \\
\text { saldrán del círculo vicioso", "tiende a acercarse a los que son apoyados en vez de los que no } \\
\text { son apoyados", "es lejano a los niños". }\end{array}$ \\
\hline $\begin{array}{l}\text { Familias } \\
\text { disfuncionales }\end{array}$ & $\begin{array}{l}\text { "son disfuncionales", "la mayoría son monoparentales", "o extendidas: mamá, papá, abuelos, } \\
\text { tíos", "el rol real de la familia es el rol patriarcal, el machismo", "la mujer tiene un rol fundamental, } \\
\text { ella es el pilar, es abnegada", "tienen completa carencia de sus roles parentales eficientes y } \\
\text { eficaces", "a veces el mismo niño se hace cargo de sí mismo", "la amenaza directa es su familia", } \\
\text { "dan la espalda a sus niños y los niños sienten el rechazo", "el hacinamiento es resultado de } \\
\text { cómo viven. Es la base de la vulnerabilidad de nuestros niños", "eso lleva a un inicio temprano } \\
\text { de la sexualidad", "escuchan lo que no deben escuchar", "ven lo que no debieran que ver o vivir", } \\
\text { "el incesto es algo común", "los propios padres o cuidadores son la amenaza directa y recae en } \\
\text { los niños tratar de salir", "hay muchas potenciales amenazas: personas mañosas, malas, que lo } \\
\text { pueden dañar, llevarlo a la perdición, a la muerte. Lo inducen a hacer cosas malas", "hay } \\
\text { violencia en los hogares". }\end{array}$ \\
\hline $\begin{array}{l}\text { - Alcohólicas y } \\
\text { drogadictas }\end{array}$ & $\begin{array}{l}\text { "viviendo juntos, el alcoholismo es esperable", "gastan entre un } 60 \text { u } 80 \% \text { del dinero para } \\
\text { recreación en alcohol y el resto en comida", "y ahora se les da todo", "les dan alimentación: } \\
\text { desayuno, almuerzo, once, incluso ropa, materiales de trabajo, útiles", "por eso la libertad que } \\
\text { ellos se dan, de dejar mucho de su dinero para tener acceso al alcohol (vino, cerveza y chicha), } \\
\text { si hay suerte". }\end{array}$ \\
\hline $\begin{array}{l}\text { - Con baja } \\
\text { escolaridad }\end{array}$ & $\begin{array}{l}\text { "muy pocos han completado } 8^{\circ} \text { básico, menos el } 4^{\circ} \text { medio", "a veces se quedan en } 5^{\circ} \text { o } 6^{\circ} \\
\text { básico", "A veces la persona cuando es la abuela o alguien que los está cuidando, tiene sexto } \\
\text { de humanidades, o incluso es analfabeta". }\end{array}$ \\
\hline Escuelas rurales & $\begin{array}{l}\text { "tienen trabajos temporales, en el campo", "si se mueve uno, se mueven todos" "los padres no } \\
\text { tienen buenos trabajos por eso se mueven de un fundo a otro", "eso hace que los niños cambien } \\
\text { mucho de colegio", “ ¿cómo los podemos educar así?" }\end{array}$ \\
\hline Escuelas urbanas & $\begin{array}{l}\text { "no les interesa el estudio", "van por cumplir", "ni siquiera asisten”, "no están interesadas en sus } \\
\text { hijos", "las familias de contextos de pobreza no están", "hay peligro de explotación infantil o que } \\
\text { no les dejen estudiar", "si la familia no apoya, cuesta lograrlo". }\end{array}$ \\
\hline $\begin{array}{l}\text { Profesor impotente } \\
\text { (al hablar de los } \\
\text { niños y las familias) }\end{array}$ & $\begin{array}{l}\text { "debemos focalizarnos en los niños que ameritan nuestros recursos y profesionalismo", "el rol } \\
\text { del profesor con alumnos apoyados por sus padres es acercarlos a la meta, motivarlos a seguir } \\
\text { adelante, a que sean mejores", "debe acercar a los niños que no tienen apoyo", "tiene que } \\
\text { hacerlo sin los padres", "el rol es de asistente social, de psicólogo", ¿cuál es su rol, llamar a los } \\
\text { papás para decirles que vayan al psicólogo, que paguen las cuentas del dividendo?" }\end{array}$ \\
\hline Profesor abrumado & $\begin{array}{l}\text { "se le están dando cada vez más responsabilidades", "está al centro, recibiendo palos de unos } \\
\text { y de otros", "no le dejan tiempo para dedicarse a sus estudiantes y llevar a la familia al trabajo", } \\
\text { "no tenemos continuidad en nuestro trabajo, por el continuo cambio de escuela de los niños". }\end{array}$ \\
\hline Los niños & $\begin{array}{l}\text { "hay niños que quieren surgir", "son iguales al resto de los niños, en lo psicológico, edad, gozan } \\
\text { de la vida, viven felices", "los niños comprometidos se agrupan, pero son menos", "no son } \\
\text { apoyados por su familia", "sufren rechazo, desinterés o los mandan a trabajar", "su vulnerabilidad } \\
\text { es producida por su familia", "ellos quieren aprender, pero sus familias no les apoyan", "la } \\
\text { vulnerabilidad la da la situación social de los niños". }\end{array}$ \\
\hline
\end{tabular}


La escuela vulnerable sería "el resultado de un poder ejercido por autoridades ineptas, ignorantes, que no tienen dirección ni conocimiento sobre la realidad pues están apartadas e incomunicadas y solo pisan y arrasan con las escuelas. Esto hace que los recursos no se usen bien y que las políticas no se apliquen porque no están bien pensadas ni entienden que esa realidad no se puede cambiar. Así, la escuela está atrapada y estancada porque requiere organización, sincronización y buena comunicación entre los actores, pero las políticas la hacen colapsar al no haber una dirección (correcta). Pero lo que más hace vulnerable a la escuela son las familias, ya que son un peligro para sus hijos. Su disfuncionalidad, la carencia de roles parentales, el hacinamiento, el alcoholismo y la drogadicción, la violencia e incestos, la baja escolaridad, su itinerancia, su falta de compromiso y de amor hacia sus hijos, hace que los niños no estén en condiciones aprender, aunque el Estado les imponga (injustamente) esta obligación a las escuelas." Y, aunque los profesores debieran hacer el cambio, ellos también impiden el logro porque son lejanos a los niños; tienen prácticas obsoletas; tienen bajas expectativas y no confían en que saldrán del círculo vicioso.

Sin embargo, estos formadores concuerdan con los formadores de la universidad tradicional sobre la visión del niño, pues es considerado igual al resto de los niños. Pero difieren en que el grupo de formadores de universidades sin requisitos de ingreso, atribuye los problemas de estos a sus propias familias. También concuerdan en la necesidad de formación del profesor, para evitar la reproducción del fracaso y del prejuicio.

\section{DISCUSIÓN}

Uno de nuestros objetivos era profundizar en las creencias sobre escuela vulnerable de los formadores de diferentes tipos de universidades. Hemos encontrado que entre los formadores de universidades sin requisitos de ingreso aparecen marcados prejuicios y creencias estereotipadas respecto a las familias de los niños. Estas creencias son comunes entre los profesores de nuestro sistema educativo (por ejemplo, ver Gómez, 2017; Apablaza, 2014; Rojas y Leyton, 2014). Según Ullucci y Howard (2015), estas creencias forman parte de la folk wisdom que se extiende ampliamente en nuestra sociedad, sin que hayan sido revisadas durante la formación, por eso, cuando los profesores aplican la etiqueta "pobre" a los niños, se convencen a sí mismos de que tienen muy poco que hacer para evitar su fracaso en la escuela, porque les falta de apoyo en el hogar o tienen padres que no valoran la educación. En esta folk wisdom se sostienen una serie de mitos: 1) Cualquiera puede salir de la pobreza, 2) Los que están en la pobreza son flojos, "parásitos del bienestar" y/o irresponsables, 3) Las personas en la pobreza comparten una "cultura" común, la cultura de la pobreza. Por otra parte, un estudio realizado en USA por Thomas-Alexander y Harper (2017) con 252 profesores mentores (un profesor supervisor que los directores seleccionan para apoyar a los estudiantes en práctica dentro de la escuela, en la última parte de su formación inicial), demuestra que estos también tenían creencias negativas semejantes a nuestros formadores de la universidad sin requisitos de ingreso, por lo que los autores sugieren que las universidades debieran seleccionar muy bien a quienes estarán a cargo de formar a los futuros profesores.

Sin duda, resolver este problema no es sencillo. La creación de políticas públicas que ponen en juego las categorías sociales que los profesores han incorporado y transmitido tan diligentemente, no harán que estos las acepten con facilidad y estén dispuestos asumir la difícil empresa de deshacer todo lo que la sociedad ha construido mediante prácticas de socialización que han encomendado a la propia escuela. Bourdieu (1999) señala que las estructuras cognitivas del mundo, no son formas de consciencia, sino que se trata de "disposiciones del cuerpo," es decir, esquemas prácticos que se han incorporado mediante prácticas sociales que están reguladas por principios implícitos que nos llaman a buscar y aceptar el orden, que ponen en funcionamiento una serie de disposiciones corporales, profundamente arraigas, que utilizamos para percibir el mundo. Esto nos lleva a entender que, para hacer posible el cambio educativo deseado, los profesores tienen que hacerse conscientes de que poseen estas disposiciones incorporadas sobre lo que es el mundo concebido como verdadero y su respectivo orden natural y llegar a tensionarlas, entender y sopesar sus consecuencias y, finalmente, desear cambiarlas. Sin embargo, Bourdieu señala que esto no se puede hacer con la simple prédica racional o la educación, no pueden cambiarse solo con tener la voluntad, "la acción simbólica no puede, por sí sola, y al margen de cualquier transformación de las condiciones de producción y fortalecimiento de las disposiciones, extirpar las creencias corporales, pasiones y pulsiones que permanecen por completo indiferentes a las conminaciones" (1999, p. 237). Si bien esto último hace parecer imposible e inútil esperar el cambio, nuestro equipo sugiere buscar estrategias suficientemente potentes que permitan intervenir en los educadores.

Paul Gorski (2016) es uno de los autores preocupados por la atribución de creencias negativas a los pobres. Gorski propone que los profesores debieran hacerse conscientes de que existen diferentes ideologías (y de que ellos también las poseen) que se utilizan como marcos interpretativos de la realidad y que estas necesitan ser revisadas cuidadosamente. Para Gorski, la visión predominante sobre la pobreza es la ideología del déficit, en la que considera que el problema se produce porque hay algo en las personas que viven en pobreza que causa su condición, tales como la actitud, la mentalidad y las deficiencias éticas, disposicionales e incluso 
espirituales de los individuos (p. 380), las que provocan su deplorable condición socioeconómica. Junto con esto, el poco involucramiento de la familia con la escuela y la baja asistencia y rendimiento de los niños, llevan fácilmente a la conclusión de que a ellos no les interesa la educación de sus hijos.

La visión más adecuada, para Gorski (2016), sería la ideología estructural, que representa una visión más compleja y crítica de esta realidad, pues considera que los bajos resultados educativos son la consecuencia de barreras estructurales, tales como la inequitativa distribución de oportunidades de acceso a la escuela y a otros recursos (vivienda inestable, baja calidad alimentaria, bajo acceso a atención de salud, falta de acceso a internet, a las artes, etc.) y son estas las que finalmente refuerzan un tipo de logro escolar en esta población. Para este autor, no hay forma de cultivar la equidad mediante ideologías evitativas como la del déficit. Según este autor, es crucial que, en primer lugar, se reconozca que los niños más pobres no rinden de la misma manera en la escuela que sus pares de niveles socioeconómicos más acomodados. Pero, a continuación, deberíamos preguntarnos cómo se puede interpretar esta situación, ¿la reconozco como inequidad? ¿soy capaz de reconocer los estereotipos y de enfrentarlos? ¿estoy dispuesto a defender la instauración de medidas impopulares para las familias más ricas, tales como eliminar las cuotas para giras de estudio, tareas que requieran tecnología o pedir a los estudiantes que relaten qué hicieron en sus "vacaciones"? Hughes (2010) señala que los programas de formación debieran cambiar la misión y las metas de la formación y crear oportunidades para el desarrollo profesional de los formadores de profesores, de modo que adquieran el conocimiento, las habilidades y la experiencia necesaria para preparar a los futuros profesores para comprender cómo aporta la escuela al mejoramiento o a la exclusión de los niños en situación de pobreza y cómo pueden evitar esto en sus aulas.

El segundo hallazgo, es que los formadores de la universidad sin requisitos de ingreso atribuyen ineptitud a sus autoridades y a los hacedores de política pública. Sin embargo, esta creencia habría que analizarla críticamente, pues está indicando el profundo descontento y malestar que presentan los profesores frente a las demandas actuales del sistema (para profundizar, revisar Rojas y Leyton, 2014). A nuestro parecer, esta puede una de las trincheras que han encontrado los profesores para poner resistencia a las políticas públicas que sienten que los perjudican, toda vez que estas incorporan sistemas de evaluación y consecuencias negativas para ellos (Rojas y Leyton, 2014; Villalobos y Quaresma, 2015).

Desde otro ángulo, algunos de los formadores de profesores de la universidad tradicional reconocen las contradicciones de las políticas públicas del sistema educativo y las distorsiones que se producen por la comprensión parcial de estas. Es importante destacar, sin embargo, que no todos los formadores de esta institución presentan esta postura. Aunque claramente sus colegas no culpabilizan a la familia ni desprecian a las autoridades, las creencias evidenciadas se refieren a problemas del sistema, de los prejuicios existentes y de la falta de oportunidades, en una forma más bien general, quedando todavía una brecha para llegar a un verdadero análisis estructural del problema. Esto no es de extrañar ya que, como se dijo en párrafos anteriores, en Chile todavía no existen instancias de trabajo colaborativo colegiadas, por lo que este pensamiento complejo compartido por algunos estos formadores, parece provenir de encuentros promovidos por los propios intereses y simpatías de estos más que por una política oficial de crear un pensamiento pedagógico común.

Ambos grupos de formadores, apuntan a la necesidad de mejoramiento en la preparación del profesor pues, en las actuales condiciones, es él quien está reproduciendo el ciclo del fracaso. No obstante, pese a este acuerdo, entre los formadores de la universidad sin requisitos de ingreso, se observa un sesgo atribucional que explica, en cierto modo, la actitud negativa de los profesores que trabajan en estas escuelas. Efectivamente, cuando hablan de las características de los niños y sus familias, aparece la imagen de un profesor agobiado e impotente ante la realidad que enfrentan y tienden a producir un discurso circular al culpar a la política pública de no conocer la realidad en que ellos se desempeñan. En esta postura se revela, además, que la identidad de estos formadores es de profesor de aula, es decir, como profesional de primer orden y no de segundo orden como debieran concebirse en este nivel educativo. Cabe señalar aquí, que la literatura refleja que los formadores que poseen menos de 5 años de experiencia, tienden a aferrarse a una identidad de profesor de aula, pero que esta cede luego de 3 a 5 años de inserción deliberada por la institución (Boyd y Harris, 2010).

Conectado con lo anterior, podríamos afirmar que las diferencias que se observan en relación a la formación de post-grado y la experiencia docente universitaria que poseen estos formadores, junto con las horas de contratación, sin duda, están influyendo en el mantenimiento de estas creencias y merecen ser discutidas con mayor profundidad. Las contrataciones por hora, si bien son rentables para las instituciones con fines de lucro, impiden el encuentro y el desarrollo de un cuerpo académico cohesionado. Pero esto no es exclusivo de estas instituciones. Como muestra Montenegro (2016), las instituciones formadoras, en general, no entregan horas para el trabajo colaborativo entre académicos, aunque cuenten con formadores con jornada completa. Lamentablemente, los mecanismos de mercado han introducido una agenda competitiva que presiona por mantener la actual desregulación (Darling-Hammond, 2016) e incentivan la reducción de costos. Si esto no 
es considerado dentro de las modificaciones que se realizan en los programas de formación de profesores, difícilmente se logrará construir una visión compartida de las metas de la formación inicial de profesores, especialmente respecto a qué pensar (creer) y cómo trabajar en escuelas de contextos de pobreza. Estos resultados podrían informar a las políticas de planta académica.

El llamado de alerta que hacemos, no pretende sugerir que solo contratando a formadores con doctorado se logrará contar con un cuerpo académico con creencias más complejas. Más bien queremos resaltar la idea de que es en este punto donde los programas de inducción, programas de desarrollo profesional y de trabajo colegiado podrían desarrollar visiones más complejas, creando espacios colaborativos que permitan reflexionar sobre cuáles son los nudos de la docencia tanto de primer como de segundo orden. $Y$ entre esos nudos, las creencias sobre la pobreza, la diversidad y muchas otras creencias relevantes, debieran ser incorporadas.

Sin duda alguna, todavía queda mucho por hacer. Se ha puesto mucho esfuerzo en mejorar lo que aprenden los estudiantes de pedagogía especialmente a través de los numerosos convenios de desempeño que ha establecido el Ministerio de Educación con las instituciones formadoras (Ávalos, 2014), pero la escasez de investigaciones sobre los formadores de profesores encargados de su educación, no nos permitía considerar que ellos también son una pieza relevante del engranaje en la reproducción del fracaso. Ahora sabemos que los formadores de profesores poseen una importante subjetividad, que les permite configurar e interpretar las realidades de las escuelas en contextos de pobreza de manera inconsciente y poco crítica. Tomando las palabras de Ullucci y Howard (2015), "los formadores de profesores deben evaluar la manera en que se prepara a los docentes para educar a los estudiantes de entornos empobrecidos y prestar mucha atención al conocimiento, los valores y las perspectivas que se les presentan" (p. 172). De otra manera, si no se atiende a las creencias que tanto formadores como estudiantes sostienen, seguiremos formando profesores que saben de antemano qué alumnos aprenderán y cuáles no lo harán, porque podrán en juego las etiquetas incorporadas y naturalizadas que responsabilizan a los niños y sus familias de su fracaso escolar. Como palabras finales, al igual que Thomas-Alexander y Harper (2017), "creemos que la escuela vulnerable que es ahora vista como un escenario negativo, podrá, algún día, con la correcta preparación y apoyo, ser vista como un lugar deseable para trabajar" (p. 63).

Las limitaciones de este estudio tienen relación con el distinto tamaño muestral de los formadores de cada tipo de institución. Por otra parte, todavía no existe un método de análisis que permita representar adecuadamente las relaciones entre el discurso y las metáforas tridimensionales construidas por los formadores. Creemos que este material tiene grandes potenciales para revelar lo que las personas piensan y creen, pero seguimos en la búsqueda de un método de interpretación integrador.

\section{CONCLUSIONES}

(1) Respecto a la caracterización de los formadores participantes en este estudio, se observan diferencias en todas las características sociodemográficas de ambos tipos de formadores: grado académico; jornada de trabajo; edad y género. Siendo los formadores de la universidad sin requisitos de ingreso los que tienen un porcentaje considerable de profesores que solo poseen licenciatura (más del 50\%) y magíster, pero ninguno con grado de doctor. Además, la mayoría con contrato por hora; con una planta más joven.

(2) Los formadores de la universidad tradicional muestran lo contrario: la mayoría posee grado de doctor y no hay formadores que solo tengan la licenciatura; la mayoría está contratado por jornada completa, tienen una edad media y hay cierto equilibrio en el porcentaje de hombres y mujeres.

(3) Los formadores de profesores de la universidad sin requisitos de ingreso revelan tener una identidad de profesor de aula, es decir, de profesional de primer orden. Esto no se manifiesta entre los formadores de la universidad tradicional.

(4) Las creencias de los formadores de universidades sin requisitos de ingreso son más prejuiciosas y estereotipadas respecto a las familias de los niños y del sistema político y de gestión, reconociéndolas como barreras insalvables que están más allá de sus manos. A su vez, las creencias de algunos formadores de profesores de la universidad tradicional reconocen las contradicciones del sistema de políticas públicas y las distorsiones que se producen por la comprensión parcial de estas, las que se expresan en exclusión y segregación. Otros formadores, en cambio, se refieren más bien a un sistema segregador y a la falta de oportunidades. Ninguno menciona a la familia como factor constituyente de la escuela vulnerable.

(5) Los dos grupos de formadores de profesores apuntan a la necesidad de mejoramiento de la preparación del profesor de aula, no existiendo programas de formación para formadores de profesores dentro de las instituciones participantes. 


\section{AGRADECIMIENTOS}

Los autores agradecen al programa FONDECYT (CONICYT) por haber financiado el proyecto № 1161721 del concurso 2016, del que nace esta publicación.

\section{REFERENCIAS}

Apablaza, M., Representaciones Sociales de Profesores Respecto de la Diversidad Escolar en Relación a los Contextos de Desempeño Profesional, Prácticas y Formación Inicial, doi: 10.4067/S0718-07052014000100001, Estudios Pedagógicos, XL(1), 7-24 (2014)

Ávalos, B., La Formación Inicial Docente en Chile: Tensiones entre Políticas de Apoyo y Control, doi: 10.4067/S071807052014000200002, Estudios Pedagógicos, XL (1), 11-28 (2014)

Boei, F. y J. Dengerink y otros cinco autores, Supporting the Professional Development of Teacher Educators in a Productive Way, doi: 10.1080/02607476.2015.1080403, Journal of Education for Teaching, 41(4), 351 -368 (2015)

Bourdieu, P., Meditaciones Pascalianas, 1를. Castellano, Anagrama, Barcelona, España (1999)

Bourdieu, P., Razones Prácticas, Sobre la Teoría de la Acción, 1a Ed., Castellano, Anagrama, Barcelona, España (1997)

Boyd, P. y K. Harris, Becoming a University Lecturer in Teacher Education: Expert School Teachers Reconstructing their Pedagogy and Identity, doi: 10.1080/19415250903454767, Professional Development in Education, 36(1-2), 9-24 (2010)

Bruner, J.J., Medio Siglo de Transformaciones de la Educación Superior Chilena: Un Estado del Arte: En La Educación Superior en Chile: Transformación, Desarrollo y Crisis, por A. Bernasconi (Ed.), 21-75, Ediciones U.C., Santiago, Chile (2015)

Borg, S., I. Clifford y K.P. Htut. Having an EfECT: Professional Development for Teacher Educators in Myanmar, doi: 10.1016/j.tate.2018.02.010, Teaching and Teacher Educator, 72, 75-86 (2018)

Cook, T.D. y C.S. Reichardt. Métodos Cualitativos y Cuantitativos en Investigación Evaluativa, 5aㅡ Ed., Morata, Madrid, España (2005)

Consejo Nacional de Educación, Índices Educación Superior, Planta Académica (2017)

Darling-Hammond, L., Research on Teaching and Teacher Education and Its Influences on Policy and Practice, doi: 10.3102/0013189X16639597, Educational Researcher, 45(2), 83-91 (2016)

Fives, H. y M. Buehl, Spring Cleaning for the "Messy" Construct of Teachers' Beliefs: What are they? Which Have Been Examined? What Can They Tell Us? in APA Educational Psychology Handbook: Individual Differences and Cultural and Contextual Factors, by K.R. Harris y otros cinco autores (Eds.), 2, 471-499, American Psychological Association, Washington D.C., USA (2012)

Gaete, A., V. Gómez y P. Benavides, The Overuse of Self-report in the Study of Beliefs in Education: Epistemological Considerations, doi:10.1080/1743727X.2017.1288205, International Journal of Research \& Method in Education, 41(3), 241-256 (2018)

Gaete, A. y J. Gaete, On Theoretical Integration in Psychotherapy, doi: 10.1037/a0038883, Journal of Psychotherapy Integration, 25(2), 158-174 (2015)

Gaete, A. y J. Gaete, El problema de la Investigación Teórica en Psicología, en Epistemología y Ciencias Sociales: Ensayos latinoamericanos, por F. Osorio (Ed.), 153-166, Ediciones LOM, Buenos Aires, Argentina (2014)

Georgiou, S., Beliefs of Experienced and Novice Teachers about Achievement, doi: 10.1080/01443410701468716, Educational Psychology: An International Journal of Experimental Educational Psychology, 28(2), 119-131 (2008)

Gómez, V., ¿Qué Creencias Sostienen Estudiantes de Pedagogía, Profesorado en Servicio y Personal Académico Formador de Docentes, en Chile, sobre la Pobreza? doi: 10.15359/ree.21-1.17, Revista Electrónica Educare, 21(1), 1-24 (2017)

González-Vallejos, M. P., El Estudio del Formador Latinoamericano: Un Campo de Investigación 'En Construcción', doi: 10.11144/Javeriana.m10-21.eflc, Magis, Revista Internacional de Investigación en Educación, 10(21), 35-54 (2018)

Gorski, P., Poverty and the Ideological Imperative: A Call to Unhook from Deficit and Grit Ideology and to strive for Structural Ideology in Teacher Education, doi: 10.1080/02607476.2016.1215546, Journal of Education for Teaching, 42(4), 378-386 (2016)

Gutiérrez, P., Exploración sobre Movilidad de Creencias Mediante Metáforas Tridimensionales Construidas en Base a la Metodología Lego $\AA^{\circledR}$ Serious Play ${ }^{\circledR}$, Tesis de Magíster, Universidad Andrés Bello, Facultad de Educación y Ciencias Sociales, Santiago de Chile (2015)

Hadar, L.L. y D. Brody, Talk about Student Learning: Promoting Professional Growth among Teacher Educators, doi: 10.1016/j.tate.2016.05.021, Teaching and Teacher Education, 59, 11-114 (2016)

Hughes, J. A., What Teacher Preparation Programs Can Do to Better Prepare Teachers to Meet the Challenges of Educating Students Living in Poverty, doi: 10.1080/01626620.2010.10463542, Action in Teacher Education, 32(1), 54-64 (2010)

James, A., Lego Serious Play; a Three-dimensional Approach to Learning Development, Journal of Learning Development in Higher Education, 6, s/p. (2013) 
Joram, E., Clashing Epistemologies: Aspiring Teachers', Practicing Teachers', and Professors' Belief about Knowledge and Research in Education, doi: 10.1016/j.tate.2006.04.032, Teaching and Teacher Education, 23, $123-135$ (2007)

Kristiansen, P. y R. Rasmussen, Building a Better Business Using the Lego Serious Play Method, John Wiley \& Sons, Hoboken, N.J, US (2014)

Levin, B.B. e Y. He, Investigating the Content and Sources of Preservice Teachers' Personal Practical Theories (PPTs), doi: 10.1177/0022487107310749, Journal of Teacher Education, 59(1), 55-68 (2008)

Loughran J., Quality in Teacher Education: Challenging Assumptions, Building Understanding through Foundation Principles; in Quality of Teacher Education and Learning. New Frontiers of Educational Research, by X. Zhu, A. Goodwin y H. Zhang (Eds.), pp. 69-84, Springer, Singapore (2017)

Loughran, J., Professionally Developing as a Teacher Educator, doi: 10.1177/0022487114533386, Journal of Teacher Education, 65(4), 271-283 (2014)

Martinez, K., Academic induction for teacher educators, doi: 10.1080/13598660701793376, Asia-Pacific Journal of Teacher Education, 36(1), 35-51 (2008)

Meckes, L. y M. Bascopé, Uneven Distribution of Novice Teachers in the Chilean Primary School System, ISSN: 10682341, Education Policy Analysis Archive, 20(30), 1-24 (2012)

Montenegro, H., The Professional Path to Become a Teacher Educator: The Experience of Chilean Teacher Educators, doi: 10.1080/19415257.2015.1051664, Professional Development in Education, 42(4), 527-546 (2016)

Murray, J. y T. Male, Becoming a Teacher Educator: Evidence from the Field, doi: 10.1016/j.tate.2004.12.006, Teaching and Teacher Education, 21(2), 125-142 (2005)

Observatorio Niñez y Adolescencia, Infancia Cuenta en Chile, Cuarto informe (2016)

Ortúzar. M. S., P. Ayala, C. Flores y C. Milesi, Percepciones acerca del Proceso de Búsqueda y Contratación de Docentes en Chile: Nudos críticos e Inequidad del Sistema, ISSN: 0717-4004, Calidad en la Educación, 45, 251.287 (2016)

Pajares, M.F., Teachers' Beliefs and Educational Research: Cleaning Up a Messy Construct, doi: 10.3102/00346543062003307, Review of Educational Research, 62(3), 307-332 (1992)

Pedraja, L., A. Araneda-Guirriman, E. Rodríguez-Ponce y J. Rodríguez-Ponce, Calidad en la Formación Inicial Docente: Evidencia Empírica en las Universidades Chilenas, doi: 10.4067/S0718-50062012000400003, Formación Universitaria, 5(4), 15-26 (2012)

Puga, I., D. Polanco y D. Corvalán, Segregación de la Formación y Carrera Docente y su Rol en la Reproducción Social de la Desigualdad, ISSN: 0717-4004, Calidad en la Educación, 43, 57-102 (2015)

Rivero, R., The Link of Teacher Career Paths on the Distribution of High Qualified Teachers: A Chilean Case Study, doi: 10.14507/epaa.v23.1710, Archivos Analíticos de Políticas Educativas, 23(73), 1-35 (2015)

Rjosk, C. y D. Richter y otros cinco autores, Socioeconomic and Language Minority Classroom Composition and Individual Reading Achievement; The Mediating Role of Instructional Quality, doi: 10.1016/j.learninstruc.2014.01.007, Language and Instruction, 32, 63-72 (2014)

Rojas, M.T. y D. Leyton, La Nueva Subjetividad Docente. Construcción de Subjetividades Docentes en los Inicios de la Implementación de la Subvención Escolar Preferencial en Chile, doi: 10.4067/S0718-07052014000200012, Estudios Pedagógicos, 40(1), 205-221 (2014)

Serbia, J. M., Diseño, Muestreo y Análisis en la Investigación Cualitativa, ISSN: 1668-5024, Hologramática, 3(7), 123-146 (2007)

Thomas-Alexander, S. y B. E. Harper, Cleaning up the Clinic: Examining Mentor Teachers' Perceptions of Urban Classrooms and Culturally Responsive Teaching, doi: 10.1515/mlt-2015-0013, Multicult. Learn. Teach, 12(1), 49-65 (2017)

Ullucci, K. e T. Howard, Pathologizing the Poor: Implications for Preparing Teachers to Work in High-Poverty Schools, doi: 10.1177/0042085914543117, Urban Education, 50(2), 170-193 (2015)

Villalobos, C. y M. L. Quaresma, Sistema Escolar Chileno: Características y Consecuencias de un Modelo Orientado al Mercado, ISSN: 1405-1435, Convergencia, Revista de Ciencias Sociales, 69, 63-84 (2015) 